\title{
Fractional-Order Adaptive Backstepping Control of a Noncommensurate Fractional-Order Ferroresonance System
}

\author{
Yan Wang $\mathbb{D},{ }^{1}$ Ling Liu, ${ }^{1}$ Chongxin Liu, ${ }^{1}$ Ziwei Zhu, ${ }^{1}$ and Zhenquan Sun ${ }^{2}$ \\ ${ }^{1}$ State Key Laboratory of Electrical Insulation and Power Equipment, School of Electrical Engineering, Xian Jiaotong University, \\ Xian 710049, China \\ ${ }^{2}$ Shaanxi Provincial Electric Power Design and Research Institute, Xian 710065, China
}

Correspondence should be addressed to Yan Wang; 1142716188@qq.com

Received 30 July 2018; Revised 3 October 2018; Accepted 29 October 2018; Published 7 November 2018

Academic Editor: S. Hassan Hosseinnia

Copyright (C) 2018 Yan Wang et al. This is an open access article distributed under the Creative Commons Attribution License, which permits unrestricted use, distribution, and reproduction in any medium, provided the original work is properly cited.

\begin{abstract}
In this paper, fractional calculus is applied to establish a novel fractional-order ferroresonance model with fractional-order magnetizing inductance and capacitance. Some basic dynamic behaviors of this fractional-order ferroresonance system are investigated. And then, considering noncommensurate orders of inductance and capacitance and unknown parameters in an actual ferroresonance system, this paper presents a novel fractional-order adaptive backstepping control strategy for a class of noncommensurate fractional-order systems with multiple unknown parameters. The virtual control laws and parameter update laws are designed in each step. Thereafter, a novel fractional-order adaptive controller is designed in terms of the fractional Lyapunov stability theorem. The proposed control strategy requires only one control input and can force the output of the chaotic system to track the reference signal asymptotically. Finally, the proposed method is applied to a noncommensurate fractional-order ferroresonance system with multiple unknown parameters. Numerical simulation confirms the effectiveness of the proposed method. In addition, the proposed control strategy also applies to commensurate fractional-order systems with unknown parameters.
\end{abstract}

\section{Introduction}

Fractional calculus is a generalization of ordinary integration and differentiation to arbitrary order [1]. In recent years, some scholars pointed out that there exist a large number of fractal phenomena in nature [2], which should not be modeled and analyzed by the classical calculus but fractional calculus. Two main outstanding advantages of fractional calculus have drawn great attention of scholars. The first advantage is that fractional calculus has unlimited memory and can take into account the previous responses up to the present time. Fractional calculus can provide clearer and more accurate description than the classical calculus methods without memory which is only a particular case. Furthermore, with the help of fractional-order calculus, we can obtain more accurate descriptions of the physical phenomena, such as electrical circuit $[3,4]$, power system $[5,6]$, signal processing [7], secure communication [8], bioengineering [9], and image encryption [10]. Second, the fractional-order $\alpha$ can enhance the flexibility of parameters and reveal various unusual characteristics of the fractionalorder system and fractional-order controllers which remain concealed in the integer-order method. It has been confirmed that the controller based on fractional calculus leads to better closed-loop performance by improving transient and steadystate responses than integer-order approaches [11, 12]. One of the most striking applications of fractional calculus is fractional-order controllers. In recent years, some excellent fractional-order controllers have been investigated, such as fractional-order PID control [13], fractional-order sliding mode control [5], fractional fuzzy control [14], and fractionalorder backstepping control [15].

As a complex nonlinear phenomenon in a power system, ferroresonance can result in chaotic oscillation and push the power system to instability, which may cause overvoltage and overcurrent, voltage collapse, and even large-scale blackout [16-18]. At present, the trend of establishing a large-scale power grid, which may cause more uncertainties 
and disturbances in the power system, results in some ferroresonance incidents. Thus, it is necessary to suppress the chaotic oscillation caused by ferroresonance and many scholars have studied some excellent methods [19-21] to eliminate ferroresonance. In fact, the actual inductance and capacitance modeled by fractional calculus are more accurate than the classical integer method [3, 4]. In addition, the fractional-order model can provide clear description of real systems with unmodeled dynamics, uncertainties, and noise, which the integer-order model fails to do. Many real dynamical circuits, like RLC circuit, resonance circuits with ultracapacitors, fractional-order Chua's circuit, and so on, have been investigated by the fractional-order model [22-26]. However, to the best of our knowledge, there are almost no reports about the fractional-order ferroresonance system, especially the more general noncommensurate case. Thus, it is necessary to investigate, study, and suppress ferroresonance by the novel and interesting fractional-order method.

Within the aforementioned fractional-order control method, the backstepping control strategy, which can simplify the controller, is an effective control technique for the systems with strict-feedback structure. Various excellent fractional-order backstepping strategies have been investigated and proposed in recent studies. In [15], a fractionalorder backstepping controller to realize the stabilization of a fractional-order chaotic system was proposed via fractional Lyapunov functions. In [27], an adaptive backstepping controller to stabilize fractional-order Chua's circuit was designed. Ref. [28] promoted the adaptive backstepping technique to the system which does not have strict-feedback structure and avoided singularity effectively in the proposed controller. In [14], an adaptive fuzzy backstepping controller was designed for the fractional-order system with unknown external disturbances. In [29], an adaptive backstepping controller was designed for the noncommensurate fractionalorder system via the fractional-order Lyapunov indirect method. In [12], a finite time fractional-order adaptive backstepping controller was applied to robotic manipulators with uncertainties and external disturbances.

In fact, the orders of actual inductance and capacitance of the ferroresonance system are not all the same which means that the fractional-order ferroresonance system is a noncommensurate fractional-order system. Nevertheless, most of the aforementioned fractional-order backstepping methods only apply to the commensurate fractional-order systems. Though the fractional-order extension of the Lyapunov direct method proposed in [30] has been used in some present works, designing an excellent controller for noncommensurate fractional-order systems is still an open and challenging problem [31]. To the best of our knowledge, there are almost no reports about noncommensurate fractional-order backstepping control for noncommensurate fractional-order via the fractional-order Lyapunov direct method. Furthermore, considering the uncertainties of a real ferroresonance system in practice, investigation of fractionalorder adaptive backstepping control for noncommensurate fractional-order systems with unknown parameters is necessary.
Motivated by the above considerations, we establish a novel noncommensurate fractional-order ferroresonance model. And then, to suppress undesirable chaotic behaviors of the proposed system, a novel fractional-order adaptive controller is designed for the noncommensurate fractionalorder system with unknown parameters via the fractionalorder Lyapunov direct method. Virtual control laws and parameter update laws are designed in terms of the backstepping procedures. The stability of the system under control is guaranteed by the fractional Lyapunov stability theorem. Finally, chaotic behaviors of the noncommensurate and commensurate fractional-order ferroresonance systems with multiple unknown parameters are eliminated, which illustrates the effectiveness and feasibility of the proposed method.

The rest of this paper is organized as follows. Section 2 contains preliminary knowledge throughout this paper. Dynamic analysis and the adaptive controller of the fractional-order ferroresonance system are presented in Section 3. Section 4 contains the simulation results. Finally, the conclusion is drawn in Section 5.

\section{Preliminary}

In the development of fractional calculus theory, many kinds of definitions were proposed. At present, the widely accepted fractional definitions are the Riemann-Liouville definition and Caputo definition. The initial value conditions of the Caputo fractional-order differential equations are clearer than the Riemann-Liouville definition. Thus, in this paper, the Caputo fractional calculus definition is employed.

Definition 1 (see [11]). The $\alpha$ th-order Caputo fractional derivative of function $f(t)$ is defined as

$$
\begin{aligned}
& { }_{t_{0}}^{C} D_{t}^{\alpha} f(t) \\
& \quad= \begin{cases}\frac{1}{\Gamma(m-\alpha)} \int_{t_{0}}^{t} \frac{f^{m}(\tau)}{(t-\tau)^{\alpha-m+1}} \mathrm{~d} \tau, & m-1<\alpha<m \\
\frac{\mathrm{d}^{m} f(t)}{\mathrm{d} t^{m}}, & \alpha=m,\end{cases}
\end{aligned}
$$

where $m$ is the smallest integer number larger than or equal to $\alpha, \Gamma(\cdot)$ is the Gamma function, and $C$ represents the Caputo definition. In this paper, ${ }_{t_{0}}^{C} D_{t}^{\alpha}$ is abbreviated as $D^{\alpha}$ when $t_{0}=$ 0 .

Properties 2 and 3 hold for both the Caputo derivative and Riemann-Liouville derivative.

Property 2 (see [2]). For $\alpha=0$, it has

$$
{ }_{0} D_{t}^{0} f(t)=f(t) .
$$

Property 3 (see [2]). The additive index law:

$$
{ }_{0} D_{t 0}^{\alpha} D_{t}^{\beta} f(t)={ }_{0} D_{t 0}^{\beta} D_{t}^{\alpha} f(t)={ }_{0} D_{t}^{\alpha+\beta} f(t) .
$$




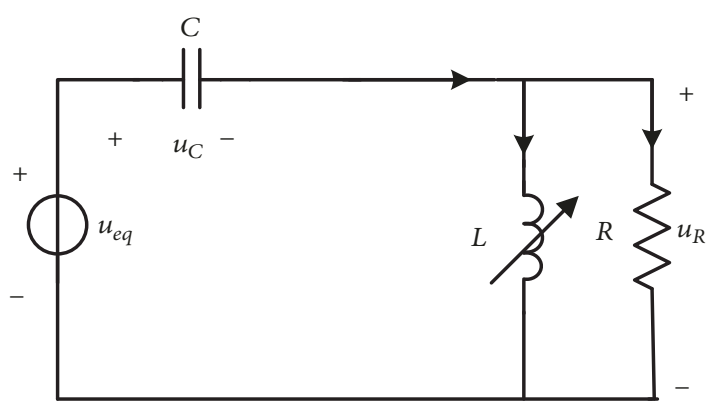

FIGURE 1: The simplified ferroresonance system circuit model.

Lemma 4 (see [32]). Let $x(t) \in \mathbb{R}$ be a continuous and derivable function. Then, for any $t \geq t_{0}$, it has

$$
\frac{1}{2}{ }_{t_{0}}^{C} D_{t}^{\alpha} x^{2}(t) \leq x(t){ }_{t_{0}}^{C} D_{t}^{\alpha} x(t), \quad \forall \alpha \in(0,1) .
$$

Lemma 5 (see [33]). Let $\boldsymbol{x}=0$ be an equilibrium point of the following nonautonomous fractional-order system:

$$
{ }_{0} D_{t}^{\alpha} \boldsymbol{x}=f(t, \boldsymbol{x})
$$

where $\alpha \in(0,1]$, the symbol $D$ can denote both the Caputo and Riemann-Liouville fractional operators, and $f(t)$ satisfies the Lipschitz condition. Assume that there exists a Lyapunov function $V(t, \boldsymbol{x}(t))$ which satisfies

$$
\begin{aligned}
\alpha_{1}(\|x\|) & \leq V(t, \boldsymbol{x}(t)) \leq \alpha_{2}(\|x\|) \\
D^{\beta} V(t, \boldsymbol{x}(t)) & \leq-\alpha_{3}(\|\boldsymbol{x}\|),
\end{aligned}
$$

where $\beta \in(0,1)$ and there exists three class $K$ functions $\alpha_{i}, i=$ $1,2,3$. Then system (5) is Mittag-Leffler stable, asymptotically.

\section{Main Results}

3.1. Dynamic Analysis. A classic two-order nonautonomous ferroresonance chaotic circuit model $[17,18]$ is shown in Figure 1. The circuit is derived by a sinusoidal voltage source $u_{e q}=\left(C_{g} /\left(C_{b}+C_{g}\right)\right) u_{S Y}$, where $C_{b}$ is the grading capacitance of circuit breaker, $C_{g}$ is the bus-to-ground capacitance, and note $C=C_{b}+C_{g}$ as equivalent capacitance. $u_{S Y}$ is the system voltage and it has $u_{S Y}=U_{m} \sin \omega t, L$ is magnetizing inductance, and $R$ is the equivalent resistance corresponding to the system losses. For very high currents, the transformer coil is saturated and the flux-current characteristic $\psi-$ $i$ of the transformer becomes highly nonlinear which is approximated by $i=a \psi+b \psi^{n}$, where $\psi$ represents the flux of the nonlinear inductance and $n$ is the index of nonlinearity of the curve.

With the help of fractional calculus, losses of actual magnetizing inductance and capacitance can be described more accurately $[3,4]$. Therefore, we try to investigate some dynamic behaviors of the ferroresonance system with noncommensurate fractional-order magnetizing inductance and capacitance. The system shown in Figure 1 can be described as

$$
\begin{aligned}
\frac{\mathrm{d}^{q_{1}} \psi}{\mathrm{d} t^{q_{1}}} & =u_{R} \\
\frac{\mathrm{d}^{q_{2}} u_{R}}{\mathrm{~d} t^{q_{2}}} & =\omega \frac{U_{m} C_{\mathrm{b}}}{C} \cos \omega t-\frac{u_{R}}{R C}-\frac{a \psi+b \psi^{n}}{C}
\end{aligned}
$$

where $q_{1}$ is the order of fractional-order magnetizing inductance $L$ and $q_{2}$ is the order of fractional-order capacitance $C$. For the general case, it usually has $q_{1} \neq q_{2}$ and $q_{1}, q_{2} \in(0,1)$. Let $x_{1}=\psi, x_{2}=u_{R} / \omega$, and $\tau=\omega t$. Then, (8) can be rewritten as

$$
\begin{aligned}
& \frac{\mathrm{d}^{q_{1}} x_{1}}{\mathrm{~d} \tau^{q_{1}}}=x_{2} \\
& \frac{\mathrm{d}^{q_{2}} x_{2}}{\mathrm{~d} \tau^{q_{2}}}=q \cos (\tau)-p x_{2}-p_{1} x-p_{2} x^{n},
\end{aligned}
$$

where $q=U_{m} C_{\mathrm{b}} / \omega C, p=1 / \omega C R, p_{1}=a / \omega^{2} C$, and $p_{2}=b / \omega^{2} C$. According to most actual $110 \mathrm{kV}$ transformer substations in China, set $p=1.155 \times 10^{-2}, p_{1}=1.94 \times 10^{-4}$, $p_{2}=4.99 \times 10^{-4}$, and $n=11$. The simulation in this paper is based on the Adams-Bashforth-Moulton method.

Remark 6. The fractional-order magnetizing inductance $L$ and fractional-order capacitance $C$ are fractance devices which can be approximately equivalent to the tree or chain structure of ideal inductance, capacitance, and resistance [34-36]. Due to the resistance of the equivalent tree or chain structure, impedance and capacitance of fractance devices contain both real and imaginary parts. The real parts will increase the loss of the fractional-order system. Furthermore, the fractance devices will result in more complicated dynamic behaviors than the integer-order model.

To investigate dynamic behaviors with different orders of the proposed system, set $q_{1}=0.99$ and $q_{2}=0.98$. As the bifurcation diagram with $q$ varying from 0 to 10 shown in Figure 2, the periodic and chaotic states appear alternately. Phase portraits and time series of states of $q=5.85$ shown in Figures 3-5 indicate that the noncommensurate fractionalorder ferroresonance system exhibits chaotic oscillation. For the integer-order case $q_{1}=q_{2}=1$, the bifurcation diagram with $q$ varying from 0 to 10 is shown in Figure 6 which indicates that the integer-order ferroresonance system exhibits chaotic oscillation with $q>0.841$. As the phase portraits and time series of states of $q=5.85$ shown in Figures 7-9, the system also exhibits chaotic oscillation. Although there exists some difference of bifurcation behaviors between Figures 2 and 6, serious overvoltage occurs in both the integer-order and noncommensurate fractionalorder case.

3.2. Controller Design. In an actual ferroresonance system, it is difficult to obtain accurate values of bus-to-ground capacitance $C_{g}$ and parameters $a$ and $b$ which results in that parameters $q, p, p_{1}$, and $p_{2}$ are actually unknown parameters. To suppress undesirable chaotic behaviors in the power 


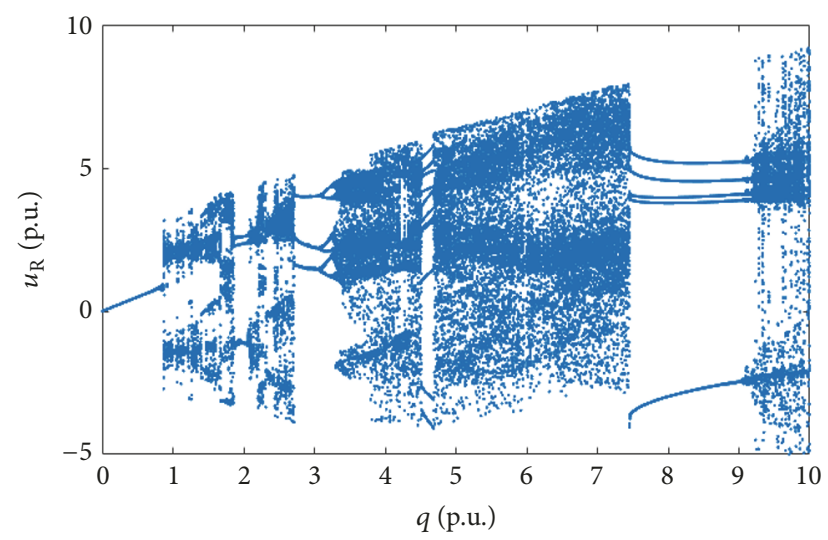

FIGURE 2: Bifurcation diagram with $q$.

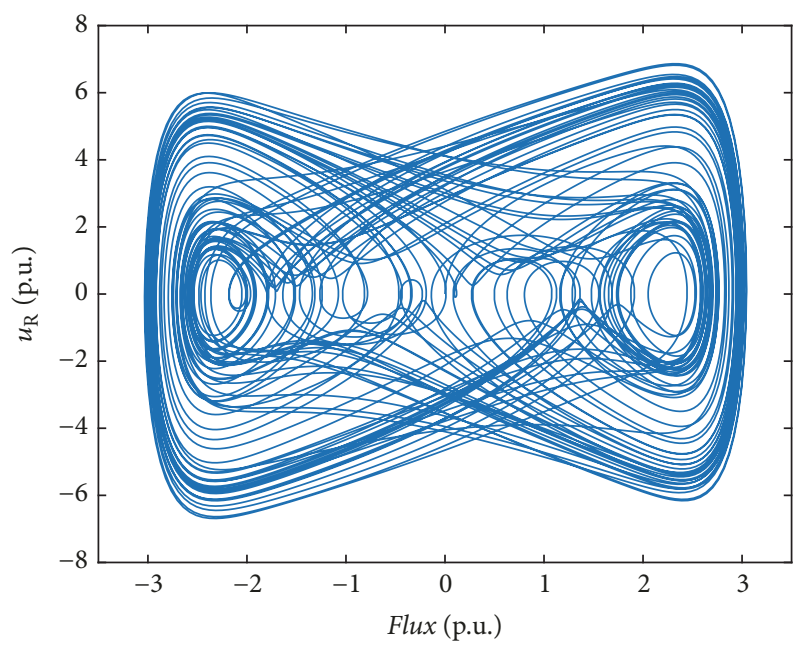

FIGURE 3: The phase portraits.

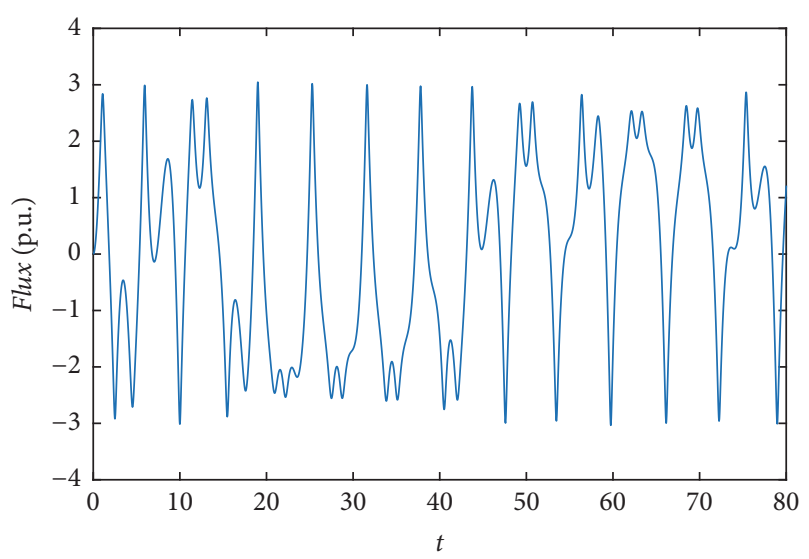

Figure 4: Time series with $x_{1}$.

system and design an effective controller for such noncommensurate fractional-order systems with multiple unknown parameters, let us consider the following strict-feedback noncommensurate fractional-order system with unknown parameters:

$$
D^{\alpha_{i}} x_{i}=c_{i} x_{i+1}+\boldsymbol{\theta}_{i}^{\mathrm{T}} \boldsymbol{F}_{i}\left(x_{1} \cdots x_{i}, t\right)+f_{i}\left(x_{1} \cdots x_{i}, t\right)
$$

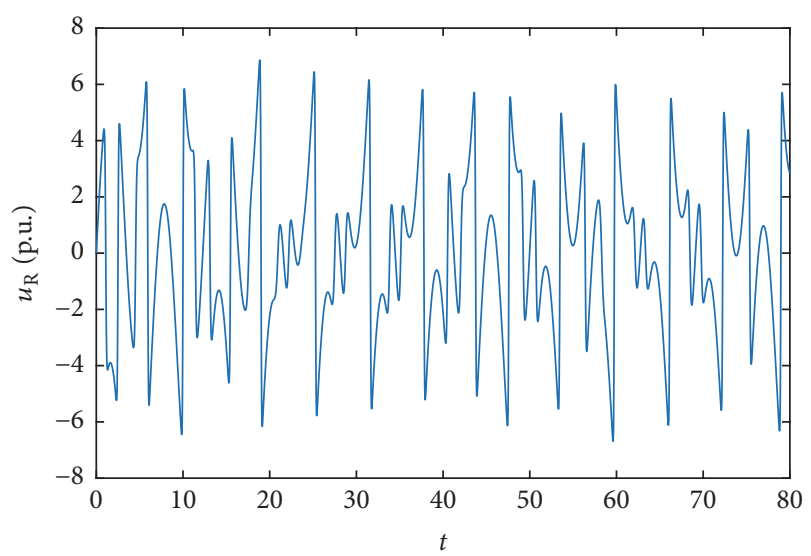

FIgURE 5: Time series with $x_{2}$.

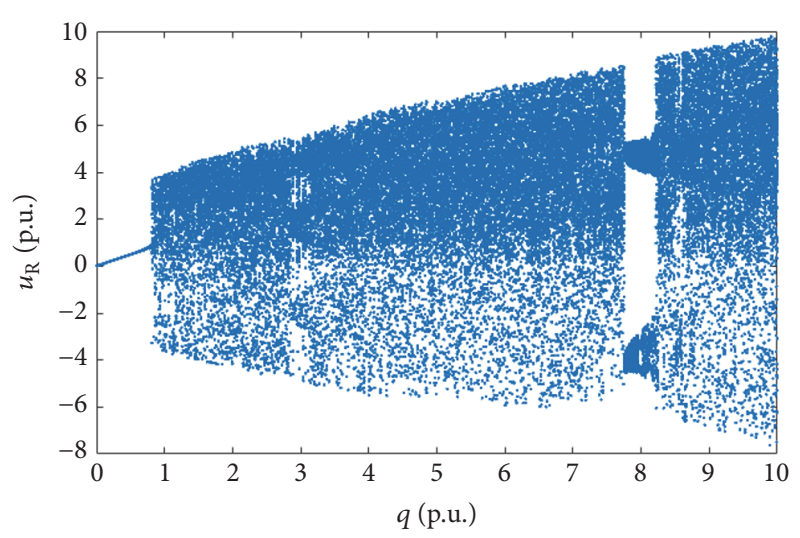

Figure 6: Bifurcation diagram with $q$.

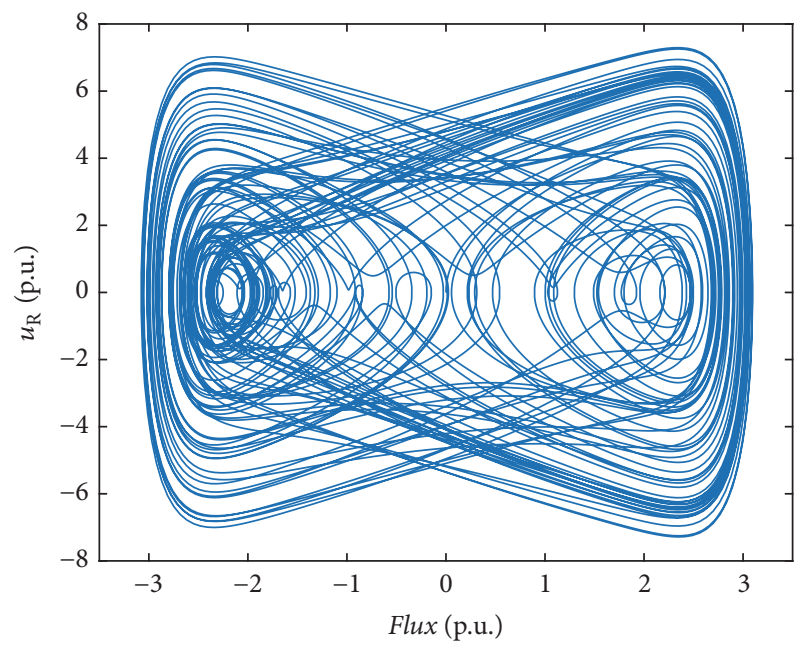

FIGURE 7: The phase portraits.

$$
\begin{array}{r}
D^{\alpha_{n}} x_{n}=c_{n} u+\boldsymbol{\theta}_{n}{ }^{\mathrm{T}} \boldsymbol{F}_{n}\left(x_{1} \cdots x_{n}, t\right)+f_{n}\left(x_{1} \cdots x_{n}, t\right) \\
y=x_{1}, i=1, \cdots, n-1,
\end{array}
$$

where system orders $\alpha_{i} \in(0,1)$ are not all the same. $\boldsymbol{x}=$ $\left[x_{1}, \cdots, x_{n}\right]^{\mathrm{T}}$ is the state vector, $u$ is controller input, and $y$ 


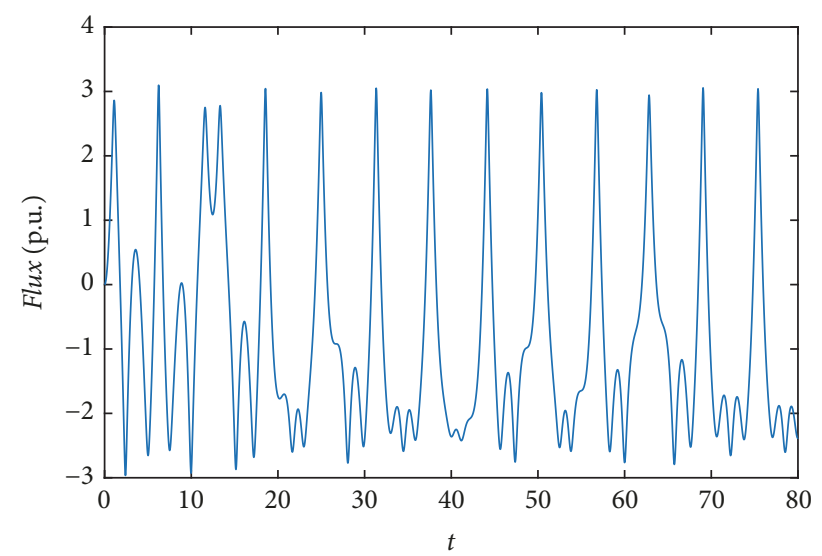

Figure 8: Time series with $x_{1}$.

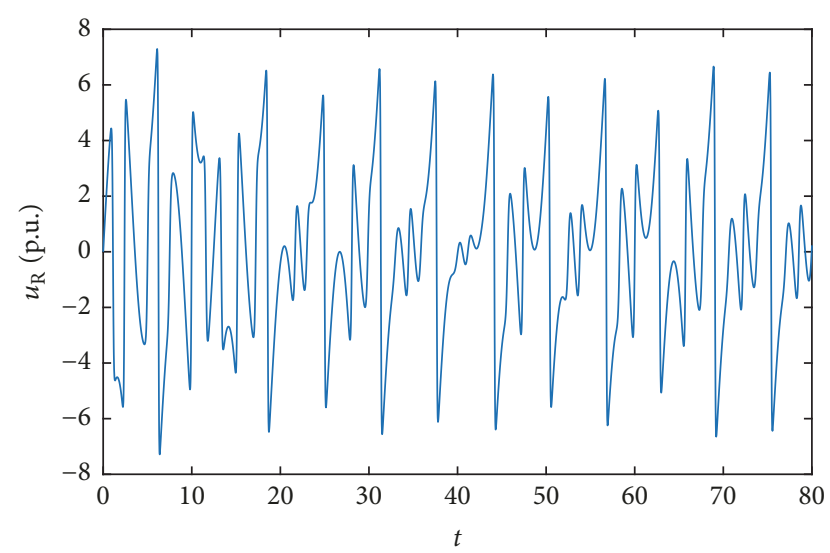

Figure 9: Time series with $x_{2}$.

is the output of system (10). $\boldsymbol{\theta}_{i} \in \mathbb{R}^{q}(i=1, \cdots, n-1)$ is the vector of unknown constant parameters. $c_{i} \in \mathbb{R}(i=1, \cdots, n)$ is a known constant. $\boldsymbol{F}_{i}\left(x_{1} \cdots x_{i}, t\right)$ and $f_{i}\left(x_{1} \cdots x_{i}, t\right)$ are known smooth nonlinear or linear functions and they are abbreviated as $\boldsymbol{F}_{i}$ and $f_{i}$ in this paper.

Obviously, noncommensurate fractional-order ferroresonance system (8) is a particular case of the strict-feedback noncommensurate fractional-order system with unknown parameters (10). To design an excellent adaptive backstepping controller for system (10), in each step, a virtual controller and parameter update law are designed systematically until the last equation which contains the controller.

The result is stated by the following theorem.

Theorem 7. For system (10), let the following designs:

The tracking error variables:

$$
e_{i}=r_{i}-x_{i}, \quad i=1,2, \cdots, n .
$$

The virtual control laws:

$$
\begin{aligned}
& r_{2}=\frac{1}{c_{1}}\left[D^{\alpha_{1}} r_{1}-\widehat{\boldsymbol{\theta}}_{1}^{\mathrm{T}} \boldsymbol{F}_{1}-f_{1}+k_{1} D^{\alpha_{1}-\alpha_{\xi}} e_{1}\right] \\
& r_{i+1}=\frac{1}{c_{i}}\left[D^{\alpha_{i}} r_{i}-\widehat{\boldsymbol{\theta}}_{i}^{\mathrm{T}} \boldsymbol{F}_{i}-f_{1}+k_{i} D^{\alpha_{i}-\alpha_{\xi}} e_{i}\right. \\
& \left.+c_{i-1} e_{i} \operatorname{sign}\left(D^{\alpha_{i-1}-\alpha_{\xi}} e_{i-1}\right) \operatorname{sign}\left(D^{\alpha_{i}-\alpha_{\xi}} e_{i}\right)\right] \\
& i=2, \cdots, n-1 .
\end{aligned}
$$

The parameter update laws:

$$
D^{\alpha_{\xi}} \hat{\boldsymbol{\theta}}_{i}^{\mathrm{T}}=-\boldsymbol{\Lambda} \cdot \boldsymbol{F}_{i} \operatorname{sign}\left(D^{\alpha_{i}-\alpha_{\xi}} e_{i}\right), \quad i=1, \cdots, n .
$$

The adaptive control law:

$$
\begin{aligned}
u= & \frac{1}{c_{n}}\left[D^{\alpha_{n}} r_{n}-\widehat{\boldsymbol{\theta}}_{n}^{\mathrm{T}} \boldsymbol{F}_{n}-f_{n}+k_{n} D^{\alpha_{n}-\alpha_{\xi}} e_{n}\right. \\
& \left.+c_{n-1} e_{n} \operatorname{sign}\left(D^{\alpha_{n-1}-\alpha_{\xi}} e_{n-1}\right) \operatorname{sign}\left(D^{\alpha_{n}-\alpha_{\xi}} e_{n}\right)\right] .
\end{aligned}
$$

Then the tracking error is stable asymptotically and globally as

$$
\lim _{t \rightarrow \infty}\left(y-r_{1}\right)=0
$$

where $\alpha_{\xi}$ is the smallest order of $\alpha_{i} . r_{1}$ is a smooth reference signal and its $\left(\alpha_{1}, \cdots, \sum_{i=1}^{n} \alpha_{i}\right)$ th-order derivatives are bounded and continuous. $k_{i}$ is a positive constant and $\boldsymbol{\Lambda}=$ $\operatorname{diag}(k, k, \cdots, k), k>0$.

Proof.

Step 1. Find the smallest order $\alpha_{\xi}$ of noncommensurate fractional-order system (10); let $e_{1}=r_{1}-x_{1}, e_{2}=r_{2}-x_{2}$. $\widetilde{\boldsymbol{\theta}}_{1}=\widehat{\boldsymbol{\theta}}_{1}-\boldsymbol{\theta}_{1}$ is the adaptive parameter tracking error and $r_{2}$ is the first virtual controller. The first subsystem is given as

$$
D^{\alpha_{1}} x_{1}=c_{1}\left(r_{2}-e_{2}\right)+\boldsymbol{\theta}_{1}^{\mathrm{T}} \boldsymbol{F}_{\mathbf{1}}+f_{\mathbf{1}} .
$$

Choose the Lyapunov function candidate as

$$
V_{1}=\left|D^{\alpha_{1}-\alpha_{\xi}} e_{1}\right|+\frac{1}{2} \widetilde{\boldsymbol{\theta}}_{1}^{\mathrm{T}} \boldsymbol{\Lambda}^{-1} \widetilde{\boldsymbol{\theta}}_{1} .
$$

Note $\mathbf{z}^{\mathrm{T}}=\left[D^{\alpha_{1}-\alpha_{\xi}} e_{1}, \widetilde{\boldsymbol{\theta}}_{1}^{\mathrm{T}}\right]$ and the K-class functions $\alpha_{1}$ and $\alpha_{2}$ are selected as $\alpha_{1}(\mathbf{z})=\min \left(|\mathbf{z}|,(1 / 2) \mathbf{z}^{\mathrm{T}} \boldsymbol{\Lambda}^{-1} \mathbf{z}\right), \alpha_{2}(\mathbf{z})=$ $|\mathbf{z}|+(1 / 2) \mathbf{z}^{\mathrm{T}} \boldsymbol{\Lambda}^{-1} \mathbf{z}$. Thus, (17) satisfies condition (6). And then, take the $\alpha_{\xi}$ order derivative of Lyapunov function (17) and use Lemma 4; we obtain

$$
\begin{aligned}
D^{\alpha_{\xi}} V_{1}= & D^{\alpha_{1}} e_{1} \cdot \operatorname{sign}\left(D^{\alpha_{1}-\alpha_{\xi}} e_{1}\right)+D^{\alpha_{\xi}}\left(\frac{1}{2} \widetilde{\boldsymbol{\theta}}_{1}^{\mathrm{T}} \boldsymbol{\Lambda}^{-1} \widetilde{\boldsymbol{\theta}}_{1}\right) \\
\leq & D^{\alpha_{1}}\left(r_{1}-x_{1}\right) \operatorname{sign}\left(D^{\alpha_{1}-\alpha_{\xi}} e_{1}\right)+\widetilde{\boldsymbol{\theta}}_{1}^{\mathrm{T}} \boldsymbol{\Lambda}^{-1} D^{\alpha_{\xi}} \widehat{\boldsymbol{\theta}}_{1} \\
\leq & \left(D^{\alpha_{1}} r_{1}+c_{1} e_{2}-c_{1} r_{2}-\boldsymbol{\theta}_{1}^{\mathrm{T}} \boldsymbol{F}_{1}-f_{1}\right) \\
& \times \operatorname{sign}\left(D^{\alpha_{1}-\alpha_{\xi}} e_{1}\right)+\widetilde{\boldsymbol{\theta}}_{1}^{\mathrm{T}} \boldsymbol{\Lambda}^{-1} D^{\alpha_{\xi}} \widehat{\boldsymbol{\theta}}_{1} .
\end{aligned}
$$

Then choose the first virtual control law $r_{2}$ as

$$
r_{2}=\frac{1}{c_{1}}\left(D^{\alpha_{1}} r_{1}-\widehat{\boldsymbol{\theta}}_{1}^{\mathrm{T}} \boldsymbol{F}_{1}-f_{1}+k_{1} D^{\alpha_{1}-\alpha_{\xi}} e_{1}\right)
$$

which leads to

$$
\begin{aligned}
D^{\alpha_{\xi}} V_{1} \leq & \left(c_{1} e_{2}+\widetilde{\boldsymbol{\theta}}_{1}^{\mathrm{T}} \boldsymbol{F}_{1}-k_{1} D^{\alpha_{1}-\alpha_{\xi}} e_{1}\right) \\
& \times \operatorname{sign}\left(D^{\alpha_{1}-\alpha_{\xi}} e_{1}\right)+\widetilde{\boldsymbol{\theta}}_{1}^{\mathrm{T}} \boldsymbol{\Lambda}^{-1} D^{\alpha_{\xi}} \widehat{\boldsymbol{\theta}}_{1} \\
\leq & -k_{1}\left|D^{\alpha_{1}-\alpha_{\xi}} e_{1}\right|+\left(c_{1} e_{2}+\widetilde{\boldsymbol{\theta}}_{1}^{\mathrm{T}} \boldsymbol{F}_{1}\right) \\
& \times \operatorname{sign}\left(D^{\alpha_{1}-\alpha_{\xi}} e_{1}\right)+\widetilde{\boldsymbol{\theta}}_{1}^{\mathrm{T}} \boldsymbol{\Lambda}^{-1} D^{\alpha_{\xi}} \widehat{\boldsymbol{\theta}}_{1} .
\end{aligned}
$$


Choose the first parameter update law $\widehat{\boldsymbol{\theta}}_{\mathbf{1}}$ as

$$
D^{\alpha_{\xi}} \widehat{\boldsymbol{\theta}}_{\mathbf{1}}=-\boldsymbol{\Lambda} \cdot \boldsymbol{F}_{1} \operatorname{sign}\left(D^{\alpha_{1}-\alpha_{\xi}} e_{1}\right) \text {. }
$$

Substitute (21) into (20), which leads to

$$
D^{\alpha_{\xi}} V_{1} \leq-k_{1}\left|D^{\alpha_{1}-\alpha_{\xi}} e_{1}\right|+c_{1} e_{2} \operatorname{sign}\left(D^{\alpha_{1}-\alpha_{\xi}} e_{1}\right) .
$$

Step 2. For the second subsystem given as

$$
D^{\alpha_{2}} x_{2}=c_{2}\left(r_{3}-e_{3}\right)+\boldsymbol{\theta}_{2}^{\mathrm{T}} \boldsymbol{F}_{2}+f_{2},
$$

choose the second Lyapunov function candidate which is similar to (17) as

$$
V_{2}=V_{1}+\left|D^{\alpha_{2}-\alpha_{\xi}} e_{2}\right|+\frac{1}{2} \widetilde{\boldsymbol{\theta}}_{2}^{\mathrm{T}} \boldsymbol{\Lambda}^{-1} \widetilde{\boldsymbol{\theta}}_{2} .
$$

Similarly, take the $\alpha_{\xi}$ order derivative of Lyapunov function (24) and use Lemma 4, which leads to

$$
\begin{aligned}
D^{\alpha_{\xi}} V_{2}= & D^{\alpha_{\xi}} V_{1}+D^{\alpha_{2}} e_{2} \cdot \operatorname{sign}\left(D^{\alpha_{2}-\alpha_{\xi}} e_{2}\right) \\
& +D^{\alpha_{\xi}}\left(\frac{1}{2} \widetilde{\boldsymbol{\theta}}_{2}^{\mathrm{T}} \Lambda^{-1} \widetilde{\boldsymbol{\theta}}_{2}\right) \\
\leq & -k_{1}\left|D^{\alpha_{1}-\alpha_{\xi}} e_{1}\right|+c_{1} e_{2} \operatorname{sign}\left(D^{\alpha_{1}-\alpha_{\xi}} e_{1}\right) \\
& +D^{\alpha_{2}}\left(r_{2}-x_{2}\right) \operatorname{sign}\left(D^{\alpha_{2}-\alpha_{\xi}} e_{2}\right) \\
& +\widetilde{\boldsymbol{\theta}}_{2}^{\mathrm{T}} \boldsymbol{\Lambda}^{-1} D^{\alpha_{\xi}} \widehat{\boldsymbol{\theta}}_{2} \\
\leq & -k_{1}\left|D^{\alpha_{1}-\alpha_{\xi}} e_{1}\right|+c_{1} e_{2} \operatorname{sign}\left(D^{\alpha_{1}-\alpha_{\xi}} e_{1}\right) \\
& +\left(D^{\alpha_{2}} r_{2}+c_{2} e_{3}-c_{2} r_{3}-\boldsymbol{\theta}_{2}^{\mathrm{T}} \boldsymbol{F}_{2}-f_{2}\right) \\
& \times \operatorname{sign}\left(D^{\alpha_{2}-\alpha_{\xi}} e_{2}\right)+\widetilde{\boldsymbol{\theta}}_{2}^{\mathrm{T}} \boldsymbol{\Lambda}^{-1} D^{\alpha_{\xi}} \widehat{\boldsymbol{\theta}}_{2} .
\end{aligned}
$$

Choose the second virtual control law $r_{3}$ as

$$
\begin{aligned}
r_{3} & =\frac{1}{c_{2}}\left[D^{\alpha_{2}} r_{2}-\widehat{\boldsymbol{\theta}}_{2}^{\mathrm{T}} \boldsymbol{F}_{2}-f_{2}+k_{2} D^{\alpha_{2}-\alpha_{\xi}} e_{2}\right. \\
& \left.+c_{1} e_{2} \operatorname{sign}\left(D^{\alpha_{1}-\alpha_{\xi}} e_{1}\right) \operatorname{sign}\left(D^{\alpha_{2}-\alpha_{\xi}} e_{2}\right)\right] .
\end{aligned}
$$

Substituting (26) into (25), we obtain

$$
\begin{aligned}
& D^{\alpha_{\xi}} V_{2} \leq-k_{1}\left|D^{\alpha_{1}-\alpha_{\xi}} e_{1}\right|+c_{1} e_{2} \operatorname{sign}\left(D^{\alpha_{1}-\alpha_{\xi}} e_{1}\right)+\left[c_{2} e_{3}\right. \\
& +\widetilde{\boldsymbol{\theta}}_{2}^{\mathrm{T}} \boldsymbol{F}_{2}-k_{2} D^{\alpha_{2}-\alpha_{\xi}} e_{2} \\
& \left.\quad-c_{1} e_{2} \operatorname{sign}\left(D^{\alpha_{1}-\alpha_{\xi}} e_{1}\right) \operatorname{sign}\left(D^{\alpha_{2}-\alpha_{\xi}} e_{2}\right)\right] \\
& \quad \times \operatorname{sign}\left(D^{\alpha_{2}-\alpha_{\xi}} e_{2}\right)+\widetilde{\boldsymbol{\theta}}_{2}^{\mathrm{T}} \boldsymbol{\Lambda}^{-1} D^{\alpha_{\xi}} \widehat{\boldsymbol{\theta}}_{2} \leq-k_{1}\left|D^{\alpha_{1}-\alpha_{\xi}} e_{1}\right| \\
& \quad-k_{2}\left|D^{\alpha_{2}-\alpha_{\xi}} e_{2}\right|+\left(c_{2} e_{3}+\widetilde{\boldsymbol{\theta}}_{2}^{\mathrm{T}} \boldsymbol{F}_{2}\right) \operatorname{sign}\left(D^{\alpha_{2}-\alpha_{\xi}} e_{2}\right) \\
& +\widetilde{\boldsymbol{\theta}}_{2}^{\mathrm{T}} \boldsymbol{\Lambda}^{-1} D^{\alpha_{\xi}} \widehat{\boldsymbol{\theta}}_{2} .
\end{aligned}
$$

Choose the second parameter update law $\widehat{\boldsymbol{\theta}}_{2}$ as

$$
D^{\alpha_{\xi}} \widehat{\boldsymbol{\theta}}_{2}^{\mathrm{T}}=-\boldsymbol{\Lambda} \cdot \boldsymbol{F}_{2} \operatorname{sign}\left(D^{\alpha_{2}-\alpha_{\xi}} e_{2}\right) .
$$

Substituting (28) into (27), we obtain

$$
\begin{aligned}
D^{\alpha_{\xi}} V_{2} \leq & -k_{1}\left|D^{\alpha_{1}-\alpha_{\xi}} e_{1}\right|-k_{2}\left|D^{\alpha_{2}-\alpha_{\xi}} e_{2}\right| \\
& +c_{2} e_{3} \operatorname{sign}\left(D^{\alpha_{2}-\alpha_{\xi}} e_{2}\right) .
\end{aligned}
$$

Step $i=(2, \cdots, n-1)$. Consider the $i$ th subsystem given as

$$
D^{\alpha_{i}} x_{i}=c_{i}\left(r_{i+1}-e_{i+1}\right)+\boldsymbol{\theta}_{i}^{\mathrm{T}} \boldsymbol{F}_{i}+f_{i} .
$$

Similarly, select the $i$ th Lyapunov function candidate as

$$
V_{i}=V_{i-1}+\left|D^{\alpha_{i}-\alpha_{\xi}} e_{i}\right|+\frac{1}{2} \widetilde{\boldsymbol{\theta}}_{i}^{\mathrm{T}} \boldsymbol{\Lambda}^{-1} \widetilde{\boldsymbol{\theta}}_{i} .
$$

Take the $\alpha_{\xi}$ order derivative of Lyapunov function (31) and use Lemma 4, which leads to

$$
\begin{aligned}
D^{\alpha_{\xi}} V_{i}= & D^{\alpha_{\xi}} V_{i-1}+D^{\alpha_{i}} e_{i} \operatorname{sign}\left(D^{\alpha_{i}-\alpha_{\xi}} e_{i}\right) \\
& +D^{\alpha_{\xi}}\left(\frac{1}{2} \widetilde{\boldsymbol{\theta}}_{i}^{\mathrm{T}} \boldsymbol{\Lambda}^{-1} \widetilde{\boldsymbol{\theta}}_{i}\right) \\
\leq & -\sum_{j=1}^{i-1}\left(k_{j}\left|D^{\alpha_{j}-\alpha_{\xi}} e_{j}\right|\right) \\
& +c_{i-1} e_{i} \operatorname{sign}\left(D^{\alpha_{i-1}-\alpha_{\xi}} e_{i-1}\right) \\
& +D^{\alpha_{i}}\left(r_{i}-x_{i}\right) \operatorname{sign}\left(D^{\alpha_{i}-\alpha_{\xi}} e_{i}\right)+\widetilde{\boldsymbol{\theta}}_{i}^{\mathrm{T}} \boldsymbol{\Lambda}^{-1} D^{\alpha_{\xi}} \widehat{\boldsymbol{\theta}}_{i} \\
\leq & -\sum_{j=1}^{i-1}\left(k_{j}\left|D^{\alpha_{j}-\alpha_{\xi}} e_{j}\right|\right) \\
& +c_{i-1} e_{i} \operatorname{sign}\left(D^{\alpha_{i-1}-\alpha_{\xi}} e_{i-1}\right) \\
& +\left(D^{\alpha_{\xi}} r_{i}+c_{i} e_{i+1}-c_{i} r_{i+1}-\boldsymbol{\theta}_{i}^{\mathrm{T}} \boldsymbol{F}_{i}-f_{i}\right) \\
& \times \operatorname{sign}\left(D^{\alpha_{i}-\alpha_{\xi}} e_{i}\right)+\widetilde{\boldsymbol{\theta}}_{i}^{\mathrm{T}} \boldsymbol{\Lambda}^{-1} D^{\alpha_{\xi}} \widehat{\boldsymbol{\theta}}_{i} .
\end{aligned}
$$

Choose the $i$ th virtual control law $r_{i+1}$ as

$$
\begin{aligned}
r_{i+1} & =\frac{1}{c_{i}}\left[D^{\alpha_{i}} r_{i}-\hat{\boldsymbol{\theta}}_{i}^{\mathrm{T}} \boldsymbol{F}_{i}-f_{i}+k_{i} D^{\alpha_{i}-\alpha_{\xi}} e_{i}\right. \\
& \left.+c_{i-1} e_{i} \operatorname{sign}\left(D^{\alpha_{i-1}-\alpha_{\xi}} e_{i-1}\right) \operatorname{sign}\left(D^{\alpha_{i}-\alpha_{\xi}} e_{i}\right)\right] .
\end{aligned}
$$

Substituting (33) into (32), we obtain

$$
\begin{aligned}
D^{\alpha_{\xi}} V_{i} \leq & -\sum_{j=1}^{i}\left(k_{j}\left|D^{\alpha_{j}-\alpha_{\xi}} e_{j}\right|\right) \\
& +\left(c_{i} e_{i+1}+\widetilde{\boldsymbol{\theta}}_{i}^{\mathrm{T}} \boldsymbol{F}_{i}\right) \operatorname{sign}\left(D^{\alpha_{i}-\alpha_{\xi}} e_{i}\right) \\
& +\widetilde{\boldsymbol{\theta}}_{i}^{\mathrm{T}} \boldsymbol{\Lambda}^{-1} D^{\alpha_{\xi}} \widehat{\boldsymbol{\theta}}_{i} .
\end{aligned}
$$


In a similar way, the $i$ th parameter update law is chosen as

$$
D^{\alpha_{\xi}} \widehat{\boldsymbol{\theta}}_{i}^{\mathrm{T}}=-\boldsymbol{\Lambda} \cdot \boldsymbol{F}_{i} \operatorname{sign}\left(D^{\alpha_{i}-\alpha_{\xi}} e_{i}\right), \quad i=2, \cdots, n-1 .
$$

Substituting (35) into (34), we obtain

$$
\begin{array}{r}
D^{\alpha_{\xi}} V_{i} \leq-\sum_{j=1}^{i}\left(k_{j}\left|D^{\alpha_{j}-\alpha_{\xi}} e_{j}\right|\right)+c_{i} e_{i+1} \operatorname{sign}\left(D^{\alpha_{i}-\alpha_{\xi}} e_{i}\right) \\
i=2, \cdots, n-1 .
\end{array}
$$

Step $n$. Finally, the overall Lyapunov function for system (10) is chosen as

$$
V_{n}=V_{n-1}+\left|D^{\alpha_{n}-\alpha_{\xi}} e_{n}\right|+\frac{1}{2} \widetilde{\boldsymbol{\theta}}_{n}^{\mathrm{T}} \boldsymbol{\Lambda}^{-1} \widetilde{\boldsymbol{\theta}}_{n}
$$

Take the $\alpha_{\xi}$ order derivative of Lyapunov function (37) and use Lemma 4 , which leads to

$$
\begin{aligned}
D^{\alpha_{\xi}} V_{n}= & D^{\alpha_{\xi}} V_{n-1}+D^{\alpha_{n}} e_{n} \operatorname{sign}\left(D^{\alpha_{n}-\alpha_{\xi}} e_{n}\right) \\
& +D^{\alpha_{\xi}}\left(\frac{1}{2} \widetilde{\boldsymbol{\theta}}_{n}^{\mathrm{T}} \boldsymbol{\Lambda}^{-1} \widetilde{\boldsymbol{\theta}}_{n}\right) \\
\leq & -\sum_{j=1}^{n-1}\left(k_{j}\left|D^{\alpha_{j}-\alpha_{\xi}} e_{j}\right|\right) \\
& +c_{n-1} e_{n} \operatorname{sign}\left(D^{\alpha_{n-1}-\alpha_{\xi}} e_{n-1}\right) \\
& +D^{\alpha_{n}}\left(r_{n}-x_{n}\right) \operatorname{sign}\left(D^{\alpha_{n}-\alpha_{\xi}} e_{n}\right) \\
& +\widetilde{\boldsymbol{\theta}}_{n}^{\mathrm{T}} \boldsymbol{\Lambda}^{-1} D^{\alpha_{\xi}} \widehat{\boldsymbol{\theta}}_{n} \\
& { }^{n-1}\left(k_{j}\left|D^{\alpha_{j}-\alpha_{\xi}} e_{j}\right|\right) \\
\leq & \sum_{j=1} \\
& +c_{n-1} e_{n} \operatorname{sign}\left(D^{\alpha_{n-1}-\alpha_{\xi}} e_{n-1}\right) \\
& +\left(D^{\alpha_{\xi}} r_{n}-c_{n} u-\boldsymbol{\theta}_{n}^{\mathrm{T}} \boldsymbol{F}_{n}-f_{n}\right) \\
& \times \operatorname{sign}\left(D^{\alpha_{n}-\alpha_{\xi}} e_{n}\right)+\widetilde{\boldsymbol{\theta}}_{n}^{\mathrm{T}} \boldsymbol{\Lambda}^{-1} D^{\alpha_{\xi}} \widehat{\boldsymbol{\theta}}_{n} .
\end{aligned}
$$

If the parameter update law and adaptive control law are designed as (13) and (14), the negative terms in (38) can be left, which leads to

$$
D^{\alpha_{\xi}} V_{n} \leq-\sum_{j=1}^{n}\left(k_{j}\left|D^{\alpha_{j}-\alpha_{\xi}} e_{j}\right|\right) \text {. }
$$

Therefore, according to Lemma 5, all the states of system (10) are globally and asymptotically stable, and tracking errors $e_{i}(i=1, \cdots, n)$ will converge to zero asymptotically under the proposed control scheme. The proof is completed.

Remark 8. The unsmooth Lyapunov function $V=\|x\|_{1}$ is selected to analyze the stability of fractional-order systems in recent literature $[5,12,37]$. In this paper, the unsmooth Lyapunov function candidate is selected as $V_{i}=V_{i-1}+$
$\left|D^{\alpha_{i}-\alpha_{\xi}} e_{i}\right|+(1 / 2) \widetilde{\boldsymbol{\theta}}_{i}^{\mathrm{T}} \boldsymbol{\Lambda}^{-1} \widetilde{\boldsymbol{\theta}}_{i}$. The term $\left|D^{\alpha_{i}-\alpha_{\xi}} e_{i}\right|$ is used to solve the problem brought by the noncommensurate orders, and the term $(1 / 2) \widetilde{\boldsymbol{\theta}}_{i}^{\mathrm{T}} \boldsymbol{\Lambda}^{-1} \widetilde{\boldsymbol{\theta}}_{i}$ can simplify the forms of parameter update laws effectively.

Remark 9. When system orders satisfy $\alpha_{i}=\alpha(i=1, \cdots$, $n$ ), system (10) becomes a strict-feedback commensurate fractional-order system with unknown parameters. It can be described as

$$
\begin{array}{r}
D^{\alpha} x_{i}=c_{i} x_{i+1}+\boldsymbol{\theta}_{i}^{\mathrm{T}} \boldsymbol{F}_{i}\left(x_{1} \cdots x_{i}, t\right)+f_{i}\left(x_{1} \cdots x_{i}, t\right) . \\
D^{\alpha} x_{n}=c_{n} u+\boldsymbol{\theta}_{n}^{\mathrm{T}} \boldsymbol{F}_{n}\left(x_{1} \cdots x_{n}, t\right)+f_{n}\left(x_{1} \cdots x_{n}, t\right) \\
y=x_{1}, i=1, \cdots, n-1
\end{array}
$$

For system (40), according to Property 2, adaptive control law (14), and parameter update laws, (13) can be rewritten as

$$
\begin{aligned}
& u= \frac{1}{c_{n}}\left[D^{\alpha} r_{n}-\widehat{\boldsymbol{\theta}}_{n}^{\mathrm{T}} \boldsymbol{F}_{n}-f_{n}+k_{n} e_{n}\right. \\
&\left.+c_{n-1} e_{n} \operatorname{sign}\left(e_{n-1}\right) \operatorname{sign}\left(e_{n}\right)\right] \\
& D^{\alpha} \widehat{\boldsymbol{\theta}}_{i}^{\mathrm{T}}=-\boldsymbol{\Lambda} \cdot \boldsymbol{F}_{i} \operatorname{sign}\left(e_{i}\right), \quad i=1, \cdots, n .
\end{aligned}
$$

For system (40), in each step, the Lyapunov function candidate is selected as

$$
V_{i}=V_{i-1}+\left|e_{i}\right|+\frac{1}{2} \tilde{\boldsymbol{\theta}}_{i}^{\mathrm{T}} \boldsymbol{\Lambda}^{-1} \widetilde{\boldsymbol{\theta}}_{i}, \quad i=2, \cdots, n .
$$

Similar to the noncommensurate case, consider system (40) with adaptive control law (41) and parameter update law (42), and take the $\alpha$ order derivative of the overall Lyapunov function $V_{n}$. Finally, it concludes as

$$
D^{\alpha} V_{n} \leq-\sum_{j=1}^{n}\left(k_{j}\left|e_{j}\right|\right) .
$$

For the commensurate fractional-order system with unknown parameters, according to Lemma 5, all the states are stable globally and tracking errors $e_{i}(i=1, \cdots, n)$ will converge to zero asymptotically under the proposed control method.

Remark 10. To illustrate the advantages of the proposed control method, the main contributions of our work are presented here as follows:

(1) More common conditions for the strict-feedback system are considered, including the noncommensurate orders, nonautonomous system, and multiple unknown parameters

(2) A novel Lyapunov function candidate $V_{i}=V_{i-1}+$ $\left|D^{\alpha_{i}-\alpha_{\xi}} e_{i}\right|+(1 / 2) \tilde{\boldsymbol{\theta}}_{i}^{\mathrm{T}} \boldsymbol{\Lambda}^{-1} \widetilde{\boldsymbol{\theta}}_{i}$ for the noncommensurate fractional-order system with unknown parameters is presented and the proposed Lyapunov function also applies to the commensurate fractional-order system

(3) Compared with existing methods, the proposed adaptive control strategy requires only one controller input which reduces the complexity and eases the implementation 


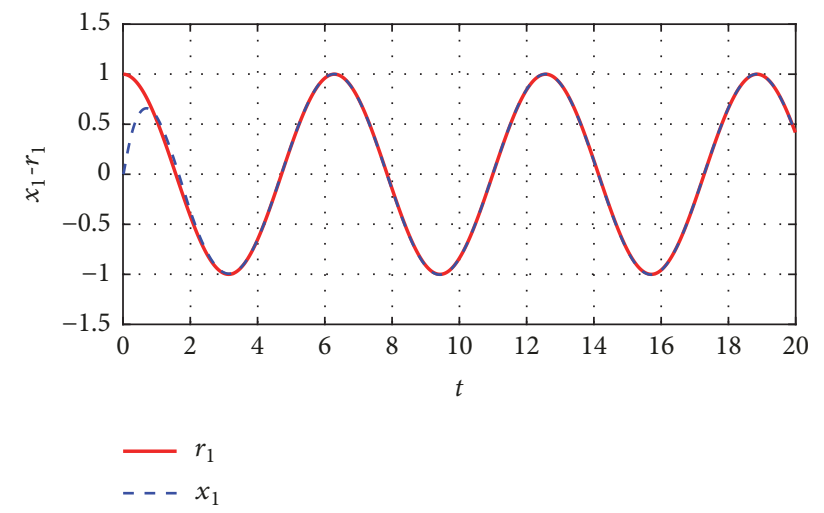

FIGURE 10: The tracking performance $x_{1}-r_{1}$ of $q_{1}=0.99$ and $q_{2}=$ 0.98 .

\section{Simulation Results}

In this section, the proposed control strategy is applied to both the noncommensurate and commensurate fractionalorder ferroresonance system with unknown parameters. The system is described as

$$
\begin{aligned}
& \frac{\mathrm{d}^{q_{1}} x_{1}}{\mathrm{~d} \tau^{q_{1}}}=x_{2} \\
& \frac{\mathrm{d}^{q_{2}} x_{2}}{\mathrm{~d} \tau^{q_{2}}}=q \cos (\tau)-p x_{2}-p_{1} x-p_{2} x^{11}+u,
\end{aligned}
$$

where $p, p_{1}, p_{2}$, and $q$ are unknown parameters and $u$ is the controller. Applying the control strategy proposed in Section 3, adaptive control law is selected as

$$
\begin{aligned}
u= & D^{q_{2}} r_{2}+k_{2} D^{q_{2}-\delta} e_{2} \\
& +e_{2} \operatorname{sign}\left(D^{q_{1}-\delta} e_{1}\right) \operatorname{sign}\left(D^{q_{2}-\delta} e_{2}\right) \\
& -\left(\widehat{p} x_{2}+\widehat{p}_{1} x_{1}+\widehat{p}_{2} x_{1}^{11}+\widehat{q} \cos \tau\right) .
\end{aligned}
$$

The parameter update laws are selected as

$$
\begin{aligned}
D^{\delta} \widehat{p} & =-k x_{2} \operatorname{sign}\left(D^{q_{2}-\delta} e_{2}\right) \\
D^{\delta} \widehat{p}_{1} & =-k x_{2} \operatorname{sign}\left(D^{q_{2}-\delta} e_{2}\right) \\
D^{\delta} \widehat{p}_{2} & =-k x_{1}^{11} \operatorname{sign}\left(D^{q_{2}-\delta} e_{2}\right) \\
D^{\delta} \widehat{q} & =-k \cos (\tau) \operatorname{sign}\left(D^{q_{2}-\delta} e_{2}\right),
\end{aligned}
$$

where $\delta$ is the smallest order between $q_{1}$ and $q_{2}, r_{1}=\cos t$ is the reference signal, $r_{2}=D^{q_{1}} r_{1}+k_{1} D^{q_{1}-\delta} e_{1}$ is the virtual control law, and $e_{1}=r_{1}-x_{1}$ and $e_{2}=r_{2}-x_{2}$ are the tracking errors. The constants are selected as $k_{1}=1.5, k_{2}=8$, and $k=3$. The initial condition is selected as $\left(x_{1}(0), x_{2}(0)\right)=$ $(0,0)$. Case 1: Tracking performance of the noncommensurate fractional-order ferroresonance system of $q_{1}=0.99$ and $q_{2}=0.98$ under control is shown in Figures 10 and 11. Case 2: Tracking performance of the commensurate fractional-order

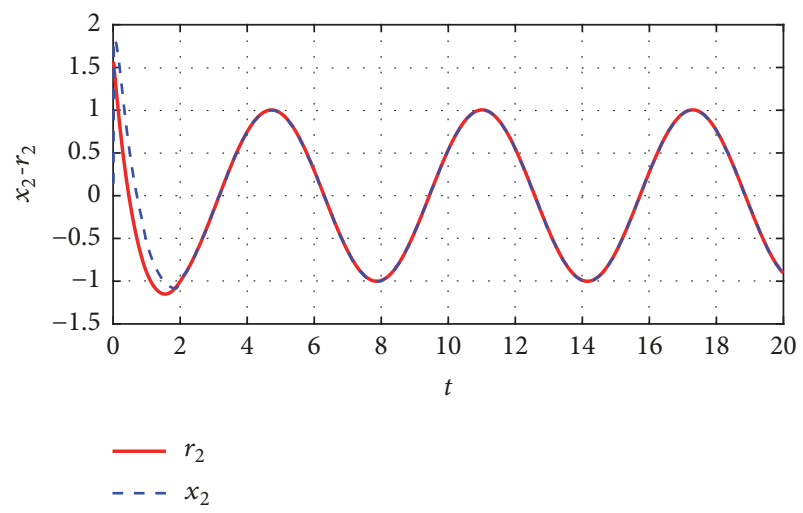

FIGURE 11: The tracking performance $x_{2}-r_{2}$ of $q_{1}=0.99$ and $q_{2}=$ 0.98 .

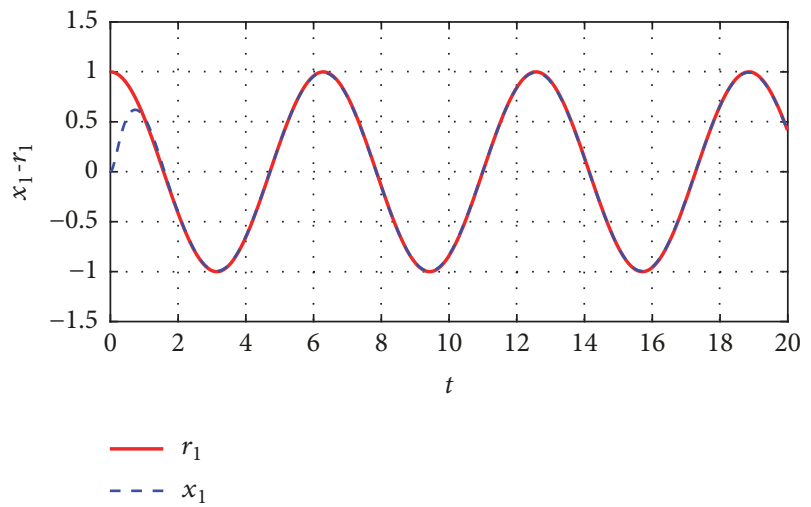

FIGURE 12: The tracking performance $x_{1}-r_{1}$ of $q_{1}=q_{2}=0.99$.

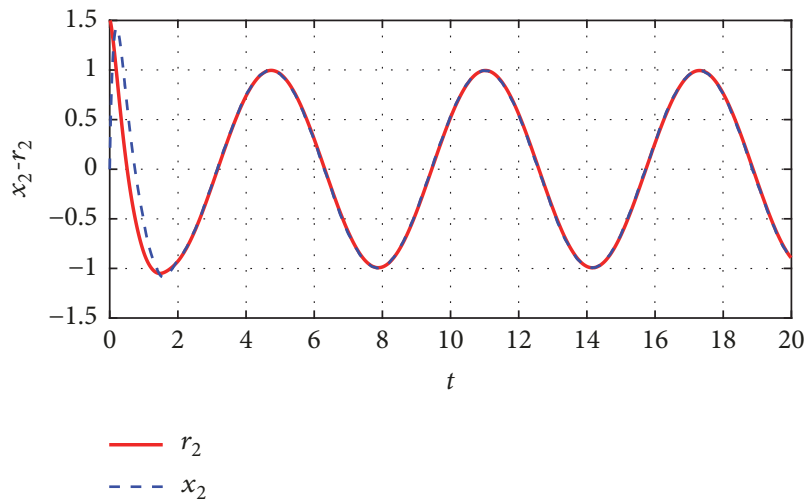

FIGURE 13: The tracking performance $x_{2}-r_{2}$ of $q_{1}=q_{2}=0.99$.

ferroresonance system of $q_{1}=q_{2}=0.99$ under control is shown in Figures 12 and 13.

From Figures 10-13, it can be seen that the state $x_{1}$ can track the reference signal $r_{1}=\cos t$ within $2 \mathrm{~s}$. Under the proposed control method, the system voltage and flux are restored to the normal level gradually. In addition, the proposed control method applies to both the noncommensurate case and commensurate fractional-order ferroresonance system with multiple unknown parameters by just one control input. 


\section{Conclusions}

In this paper, fractional calculus is applied to investigate the dynamic behaviors of a ferroresonance system with noncommensurate fractional-order magnetizing inductance and capacitance. The simulations illustrate that chaotic oscillation could occur in both the integer-order and noncommensurate fractional-order case. Considering the unknown parameters of a real system and the different orders of actual magnetizing inductance and capacitance, a novel fractional-order adaptive backstepping control method is proposed to suppress chaotic behaviors of noncommensurate fractional-order strict-feedback chaotic systems with unknown parameters via the fractional-order Lyapunov direct method. Parameter update laws and virtual control laws are designed in each step. And then, a novel adaptive backstepping controller is designed to force the system output to track the reference signal. Asymptotic stability of the fractional-order chaotic system under control is guaranteed by the fractional Lyapunov stability theorem. Compared with existing methods, the proposed control scheme not only applies to the noncommensurate case, but also requires just one control input which can simplify implementation in practice. Finally, the proposed control strategy is applied to the noncommensurate fractional-order ferroresonance system with unknown parameters. Simulation results demonstrate the effectiveness of the proposed method. In addition, it is worth noting that the proposed method also applies to the commensurate fractional-order ferroresonance system with unknown parameters.

\section{Data Availability}

The data used to support the findings of this study are included within the article.

\section{Conflicts of Interest}

There are no conflicts of interest regarding the publication of this paper.

\section{Acknowledgments}

This project was supported by the National Natural Science Foundation of China (Grant No. 51877162) and the Science Fund for Creative Research Groups of the National Natural Science Foundation of China (Grant No. 51521065).

\section{References}

[1] M. S. Tavazoei, M. Haeri, S. Jafari, S. Bolouki, and M. Siami, "Some applications of fractional calculus in suppression of chaotic oscillations," IEEE Transactions on Industrial Electronics, vol. 55, no. 11, pp. 4094-4101, 2008.

[2] I. Petráš, Fractional-Order Nonlinear Systems: Modeling, Anal$y$ sis and Simulation, Higher Education Press, Beijing, China, 2011.

[3] S. Westerlund and L. Ekstam, "Capacitor theory," IEEE Transactions on Dielectrics and Electrical Insulation, vol. 1, no. 5, pp. 826-839, 1994.
[4] I. Schäfer and K. Krüger, "Modelling of lossy coils using fractional derivatives," Journal of Physics D: Applied Physics, vol. 41, no. 4, 2008.

[5] J. Ni, L. Liu, C. Liu, and X. Hu, "Fractional order fixed-time nonsingular terminal sliding mode synchronization and control of fractional order chaotic systems," Nonlinear Dynamics, vol. 89, no. 3, pp. 2065-2083, 2017.

[6] W. Zheng, Y. Luo, Y. Chen, and Y. Pi, "Fractional-order modeling of permanent magnet synchronous motor speed servo system," Journal of Vibration and Control, vol. 22, no. 9, pp. 2255-2280, 2016.

[7] S.-C. Pei and J.-J. Ding, "Relations between Gabor transforms and fractional Fourier transforms and their applications for signal processing," IEEE Transactions on Signal Processing, vol. 55, no. 10, pp. 4839-4850, 2007.

[8] I. N'Doye, H. Voos, and M. Darouach, "Observer-based approach for fractional-order chaotic synchronization and secure communication," IEEE Journal on Emerging and Selected Topics in Circuits and Systems, vol. 3, no. 3, pp. 442-450, 2013.

[9] R. L. Magin, "Fractional calculus in bioengineering: A tool to model complex dynamics," in Proceedings of the 13th International Carpathian Control Conference (ICCC '12), pp. 464-469, May 2012.

[10] J. Hou, R. Xi, P. Liu, and T. Liu, "The switching fractional order chaotic system and its application to image encryption," IEEE/CAA Journal of Automatica Sinica, vol. 4, no. 2, pp. 381388, 2017.

[11] I. Petráš, Fractional Differential Equations, Academic Press, New York, NY, USA, 1999.

[12] N. Nikdel, M. Badamchizadeh, V. Azimirad, and M. A. Nazari, "Fractional-Order Adaptive Backstepping Control of Robotic Manipulators in the Presence of Model Uncertainties and External Disturbances," IEEE Transactions on Industrial Electronics, vol. 63, no. 10, pp. 6249-6256, 2016.

[13] A. Dumlu and K. Erenturk, "Trajectory tracking control for a 3-DOF parallel manipulator using fractional-order $\mathrm{PI}^{\lambda} \mathrm{D}^{\mu}$ control," IEEE Transactions on Industrial Electronics, vol. 61, no. 7, pp. 3417-3426, 2014.

[14] H. Liu, Y. Pan, S. Li, and Y. Chen, "Adaptive Fuzzy Backstepping Control of Fractional-Order Nonlinear Systems," IEEE Transactions on Systems, Man, and Cybernetics: Systems, vol. 47, no. 8, pp. 2209-2217, 2017.

[15] M. K. Shukla and B. B. Sharma, "Backstepping based stabilization and synchronization of a class of fractional order chaotic systems," Chaos, Solitons \& Fractals, vol. 102, pp. 274-284, 2017.

[16] C. Charalambous, Z. D. Wang, M. Osborne, and P. Jarman, "Sensitivity studies on power transformer ferroresonance of a $400 \mathrm{kV}$ double circuit," IET Generation, Transmission \& Distribution, vol. 2, no. 2, pp. 159-166, 2008.

[17] S. Mozaffari, M. Sameti, and A. C. Soudack, "Effect of initial conditions on chaotic ferroresonance in power transformers," IEE Proceedings - Generation, Transmission and Distribution, vol. 144, no. 5, pp. 456-460, 1997.

[18] A. E. A. Araujo, A. C. Soudack, and J. R. Marti, "Ferroresonance in power systems: chaotic behaviour," IEE Proceedings Part C Generation, Transmission and Distribution, vol. 140, no. 3, pp. 237-240, 1993.

[19] S.-J. Huang and C.-H. Hsieh, "Relation analysis for ferroresonance of bus potential transformer and circuit breaker grading capacitance," International Journal of Electrical Power \& Energy Systems, vol. 51, no. 3, pp. 61-70, 2013. 
[20] M. Yang, W. Sima, Q. Yang, J. Li, M. Zou, and Q. Duan, "Non-linear characteristic quantity extraction of ferroresonance overvoltage time series," IET Generation, Transmission \& Distribution, vol. 11, no. 6, pp. 1427-1433, 2017.

[21] S. Rezaei, "Adaptive overcurrent protection against ferroresonance," IET Generation, Transmission \& Distribution, vol. 12, no. 7, pp. 1573-1588, 2018.

[22] J. Walczak and A. Jakubowska, "Resonance in series fractional order $R L_{\beta} C_{\alpha}$ circuit," Przeglad Elektrotechniczny, vol. 4, pp. 210213, 2014.

[23] J. Walczak and A. Jakubowska, "Analysis of resonance phenomena in series RLC circuit with supercapacitor," Lecture Notes in Electrical Engineering: Analysis and Simulation of Electrical and Computer System, vol. 324, pp. 27-34, 2015.

[24] A. Jakubowska-Ciszek and J. Walczak, "Analysis of the transient state in a parallel circuit of the class $R L_{\beta} C_{\alpha}$ " Applied Mathematics and Computation, vol. 319, pp. 287-300, 2018.

[25] I. Petrá, "A note on the fractional-order Chuas system," Chaos, Solitons \& Fractals, vol. 38, no. 1, pp. 140-141, 2008.

[26] D. Sierociuk, G. Sarwas, and M. Twardy, "Resonance phenomena in circuits with ultracapacitors," in Proceedings of the 12th International Conference on Environment and Electrical Engineering (EEEIC '13), pp. 197-202, Wroclaw, Poland, May 2013.

[27] M. K. Shukla and B. B. Sharma, "Stabilization of a class of uncertain fractional order chaotic systems via adaptive backstepping control," in Proceedings of the 3rd Indian Control Conference (ICC '17), pp. 462-467, Guwahati, India, January 2017.

[28] M. K. Shukla and B. B. Sharma, "Stabilization of a class of fractional order chaotic systems via backstepping approach," Chaos, Solitons \& Fractals, vol. 98, pp. 56-62, 2017.

[29] Y. Wei, P. W. Tse, D. Sheng, and Y. Wang, "Fractional order adaptive backstepping output feedback control: The incommensurate case," in Proceedings of the 32nd Youth Academic Annual Conference of Chinese Association of Automation (YAC '17), pp. 366-372, Hefei, China, May 2017.

[30] Y. Li, Y. Chen, and I. Podlubny, "Stability of fractional-order nonlinear dynamic systems: Lyapunov direct method and generalized Mittag-Leffler stability," Computers \& Mathematics with Applications, vol. 59, no. 5, pp. 1810-1821, 2010.

[31] M. P. Aghababa, "Stabilization of a class of fractional-order chaotic systems using a non-smooth control methodology," Nonlinear Dynamics, vol. 89, no. 2, pp. 1357-1370, 2017.

[32] N. Aguila-Camacho, M. A. Duarte-Mermoud, and J. A. Gallegos, "Lyapunov functions for fractional order systems," Communications in Nonlinear Science and Numerical Simulation, vol. 19, no. 9, pp. 2951-2957, 2014.

[33] Y. Li, Y. Chen, and I. Podlubny, "Mittag-Leffler stability of fractional order nonlinear dynamic systems," Automatica, vol. 45, no. 8, pp. 1965-1969, 2009.

[34] M. Nakagawa and K. Sorimachi, "Basic characteristics of a fractance device," IEICE Transactions on Fundamentals of Electronics, Communications and Computer Sciences, vol. 75, pp. 1814-1819, 1992.

[35] G. E. Carlson and C. A. Halijak, "Approximation of fractional capacitors (1/s)1/n by a regular Newton process," IEEE Transactions on Circuit Theory, vol. 11, no. 2, pp. 210-213, 1964.

[36] Z. Jia and C. Liu, "Fractional-order modeling and simulation of magnetic coupled boost converter in continuous conduction mode," International Journal of Bifurcation and Chaos, vol. 28, no. $05,2018$.
[37] M. P. Aghababa, "Stabilization of a class of fractional-order chaotic systems using a non-smooth control methodology," Nonlinear Dynamics, vol. 69, no. 1-2, pp. 247-261, 2012. 


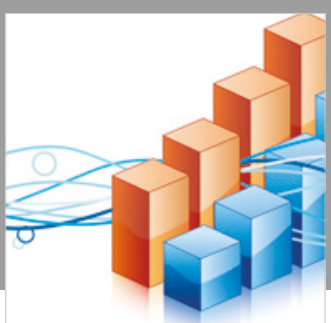

Advances in

Operations Research

\section{-n-m}
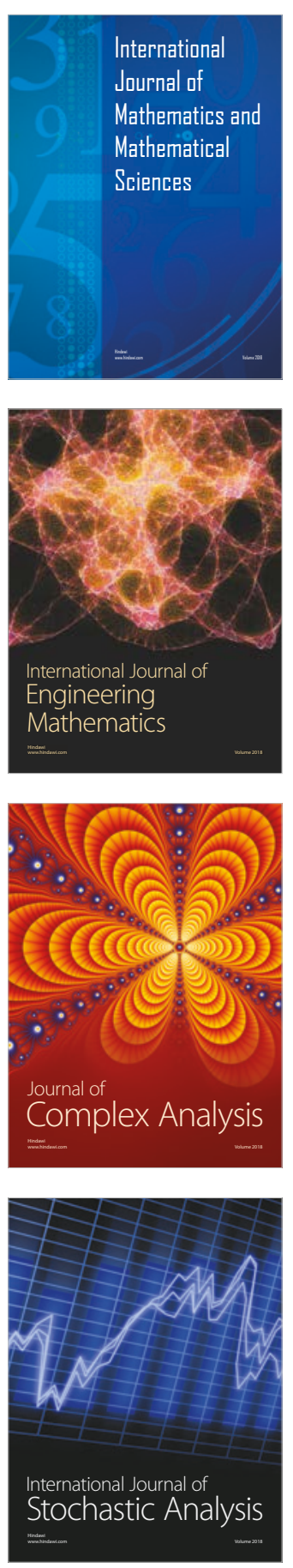
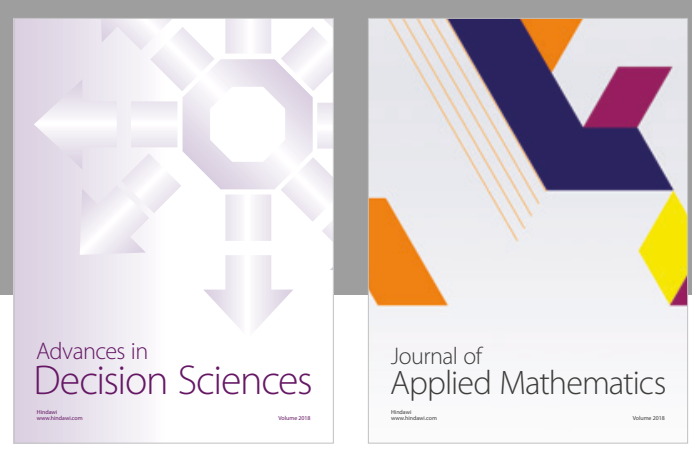

Journal of

Applied Mathematics
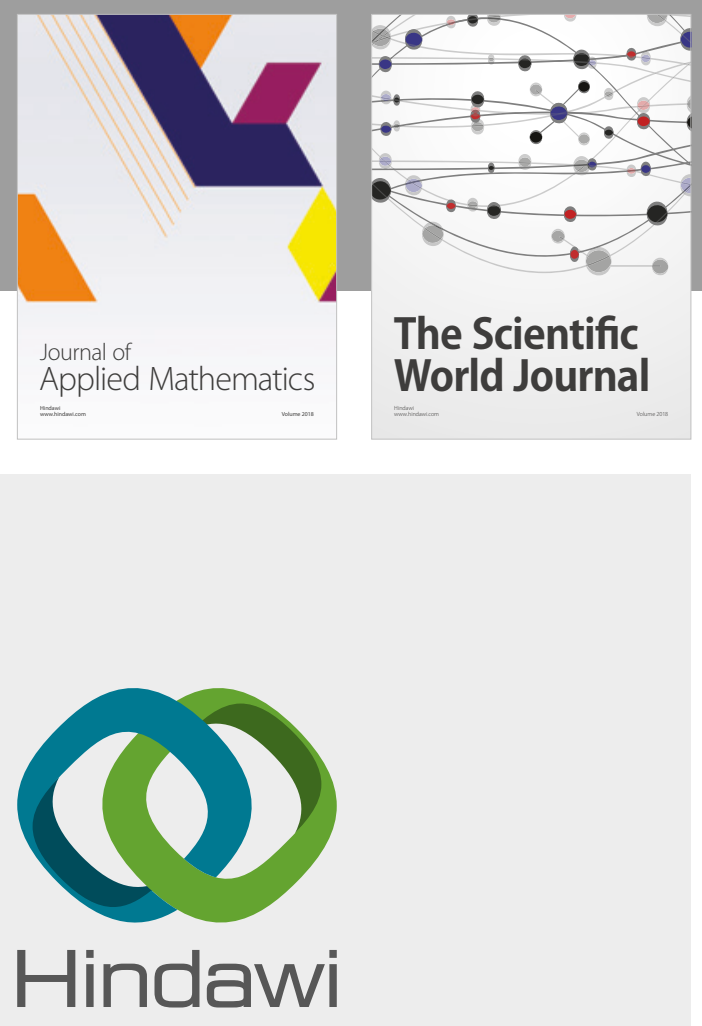

Submit your manuscripts at

www.hindawi.com

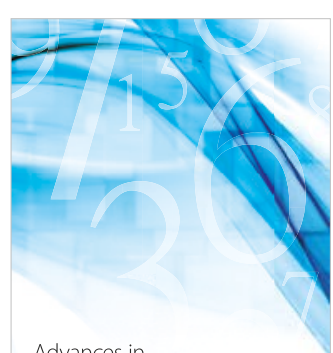

Advances in
Numerical Analysis
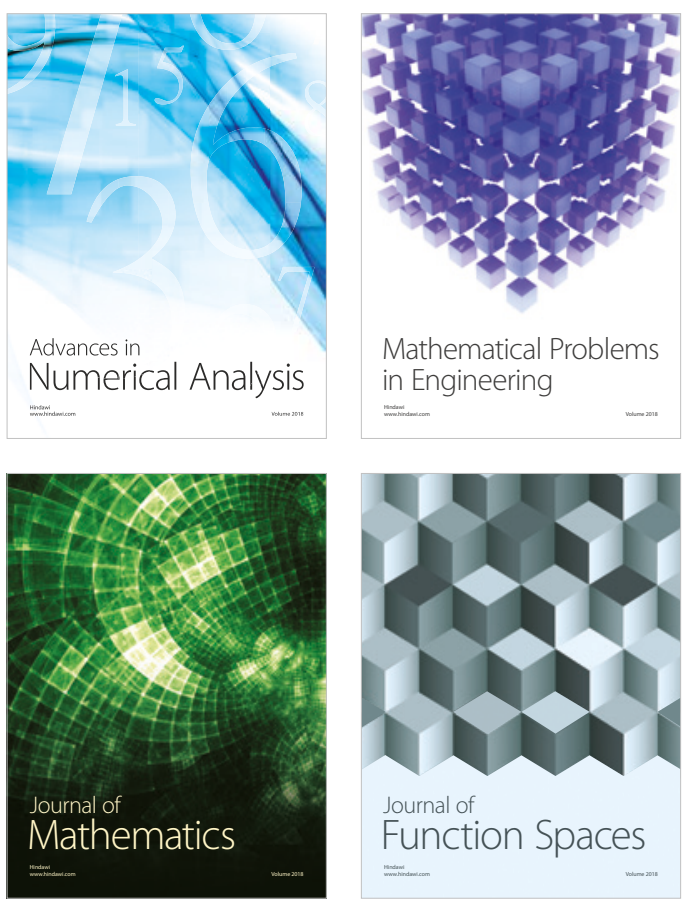

Mathematical Problems in Engineering

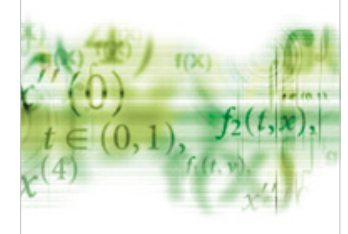

International Journal of

Differential Equations

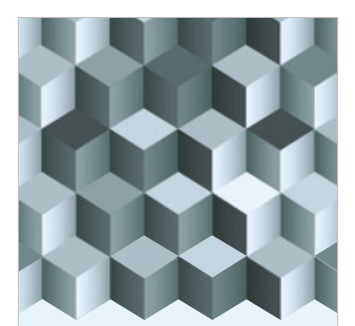

Journal of

Function Spaces

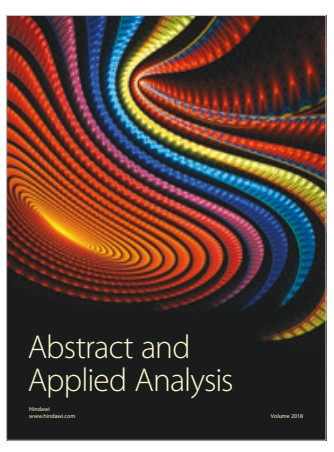

The Scientific

World Journal

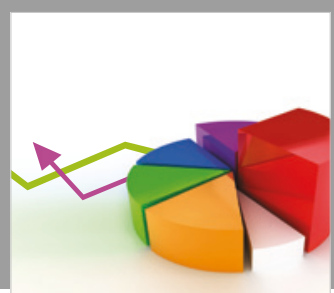

Journal of

Probability and Statistics
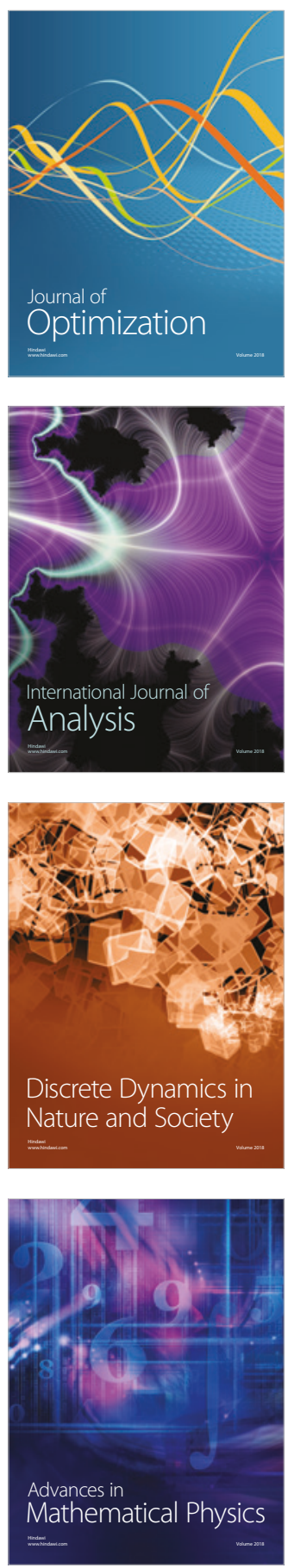